\title{
Optimized 3D and true-azimuth internal multiple attenuation strategy in the Santos Basin
}

Frederico Xavier de Melo*1, Marina Fagundes Pantoja ${ }^{1}$, Marcela Ortin ${ }^{1}$, Joel Monteiro ${ }^{1}$,Elena Medina ${ }^{1}$, ${ }^{1}$ Schlumberger,

Copyright 2021, SBGf - Sociedade Brasileira de Geofísica

This paper was prepared for presentation during the $17^{\text {th }}$ International Congress of the Brazilian Geophysical Society held in Rio de Janeiro, Brazil, 16-19 August 2021.

Contents of this paper were reviewed by the Technical Committee of the $17^{\text {th }}$ International Congress of the Brazilian Geophysical Society and do not necessarily represent any position of the SBGf, its officers or members. Electronic reproduction or storage of any part of this paper for commercial purposes without the written consent of the Brazilian Geophysical Society is prohibited.

\section{Abstract}

The Santos Basin is one of the largest Brazilian sedimentary basins and the location of several discoveries of oil and gas fields. The basin is known for its geological complexity, with the main reservoir placed over $5000 \mathrm{~m}$ below the seafloor under a complex overburden. The setting is composed of structural trend variations and evaporites with variable thickness, in addition to the presence of igneous layers mixed with sandstone, shale, and salt. This type of geological complexity creates the perfect condition for strong internal multiple contamination at the reservoir level. This work shows a three-pronged approach focused on internal multiple attenuation during the velocity model building and final imaging processes.

\section{Introduction}

Interest in deep-water offshore exploration with focus towards deeper and complex reservoir targets, especially below salt structures, has increased the awareness of the importance of addressing coherent noise related to seismic wavefield scattering effects at the overburden levels. These conditions increase the need for methods and workflow development targeting the attenuation of such interference.

Complex stratigraphy with high acoustic contrasts, beginning at the seafloor down to deeper reservoir levels, creates the ideal condition for strong internal multiples. Small-scale geological features (e.g., igneous rock intrusions) can also act as generators for diffracted internal multiples. These types of multiples obscure primary reflection information from the seismic data, making earth model building, interpretation, and inversion ambiguous at the producing target zones. One may experience scenarios ranging from clear and interpretable internal multiples arriving inside the crystalline salt body structure, to a relatively weak, proximal and conformable trend of multiples perversely interacting with primaries.

Data-driven prediction methods based on the selection of recorded sub-events at the sea surface are effective approaches when it comes to internal multiple attenuation (IMA). Current state-of-the-art algorithms perform under different frameworks, such as boundary-related (Jakubowicz, 1998; Verschuur, 2006; Wu et al., 2011), Marchenko-based (Van der Neut, 2016), or methods based on a leading term of the inverse scattering series (Weglein et al., 1997).

A complete data-driven internal multiple attenuation workflow consists of the prediction followed by the adaptive matching step. Recent approaches, focused on complex deep-water environments, performed the prediction in the data domain, with the adaptive-matching operation postponed as a post-imaging process. The main reason behind this decision relates to the overall complexity of the wavefield at deeper targets. The imaging process simplifies the adaptive matching operation, coherently positioning the recorded data and predicted model. Although this might be an acceptable approach, it still requires an accurate velocity model in place, which might not be the case for seismic experiments in the exploration phase of the development of a field.

In this work, we propose a three-pronged approach addressing the internal multiple contamination over a seismic exploration project in the Santos Basin, offshore Brazil as follows, and as shown in Figure 1:

-Optimized 3D data-driven prediction of internal multiples using interface-related generator method;

-Data-domain internal multiple attenuation as input for deep reflection-based full-waveform inversion (RFWI);

-Final adaptive matching of internal multiples performed after imaging of the recorded data and predicted model using the updated velocity field.

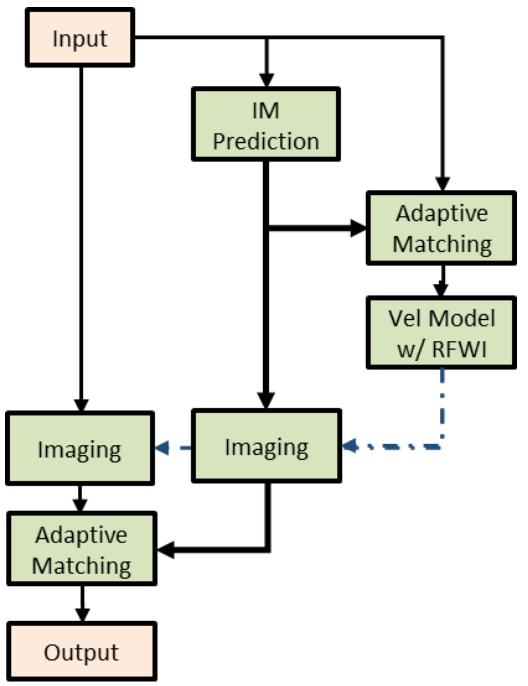

Figure 1 - Overview of the internal multiple attenuation worfklow. 


\section{Method}

\section{$3 D$ data-driven internal multiple prediction}

The boundary-based method was originally proposed by Jakubowicz (1998) and predicts all internal multiples with a common downward reflection at a given interpreted interface or horizon. Verschuur (2006) and Wu et al. (2011) discuss practical aspects and implementations. This work considered a sequence of variable and overlapping interfaces as generator components, capturing all internal multiples generated downward from the seafloor until the pre-salt formations.

Internal multiple prediction occurs by a set of convolution and cross-correlation operations using a set of three traces (source, receiver and generator component traces referred to as triplets), where all possible combinations over a surface grid (aperture) for downward reflection points (DRPs) are considered as part of the multiple contribution gather (MCG). This operation is performed for each generating interface defined in the prediction process. For complex geology commonly found in the Santos Basin, the required computation of all DRPs within the MCG is a very intense process, often resulting in prohibitive computational costs in the signal processing project. We used a new optimized approach when computing the triplets (Wu et al., 2019) to provide an accurate model in complex geological environments while capturing the full 3D effect of the internal multiple wavefield. Figure 2 shows a simple schematic of the computation of an internal multiple $\overline{\mathbf{I M}}$ governed by:

$\overline{\operatorname{IM}}_{\left(\mathrm{x}_{\mathrm{s}} \mathrm{y}_{\mathrm{s}} ; \mathrm{x}_{\mathrm{T}} \mathrm{y}_{\mathrm{T}}\right)}^{\mathrm{gk}}=$

$\frac{1}{A^{\prime}} \iiint \tilde{\mathbf{S}}_{\left(x_{s} y_{s} ; x_{i} y_{i}\right)}^{k} \circledast \widetilde{\mathbf{R}}_{\left(x_{j} y_{i} ; x_{T} y_{T}\right)}^{k} \otimes \widetilde{\mathbf{G}}_{\left(x_{i} y_{i} ; x_{j} y_{i}\right)}^{k} d x_{i} d x_{j} d y_{i}$

Where $\widetilde{\mathbf{S}}, \widetilde{\mathbf{R}}, \widetilde{\mathbf{G}}$ are sources, receivers and generator component traces, mapped over spatial coordinates $(x, y)$ for each k-th generator. Summation limits and indices $(i, j)$ define the overall aperture and DRP sampling of a given MCG, normalized by $A^{\prime}$ after the stacking process. This operation is performed for a given number of traces within the seismic experiment, honoring the original acquisition geometry (offset and azimuth) pertinent to the project. Post-conditioning applied to modeled multiples compensates for eventual transmission and reflection losses coming from deeper interfaces present at the generator component, but not experienced by the recorded internal multiple.

\section{Internal multiple attenuation in the data domain as pre- processing for pre-salt velocity model building}

Velocity models at pre-salt prospect levels are difficult to update due of illumination challenges related to overburden complexity. RFWI algorithms overcome these obstacles by producing accurate low-wavenumber model updates (Sun et al., 2018). Based on a Born modelingbased gradient kernel and robust kinematics-oriented objective functions, these methods assume that only primary reflections are present in the recording. The presence of strong internal multiples at deep and complex pre-salt zones negatively affects the velocity modelbuilding processes, ultimately impairing the final imaging outcome at the reservoir levels.

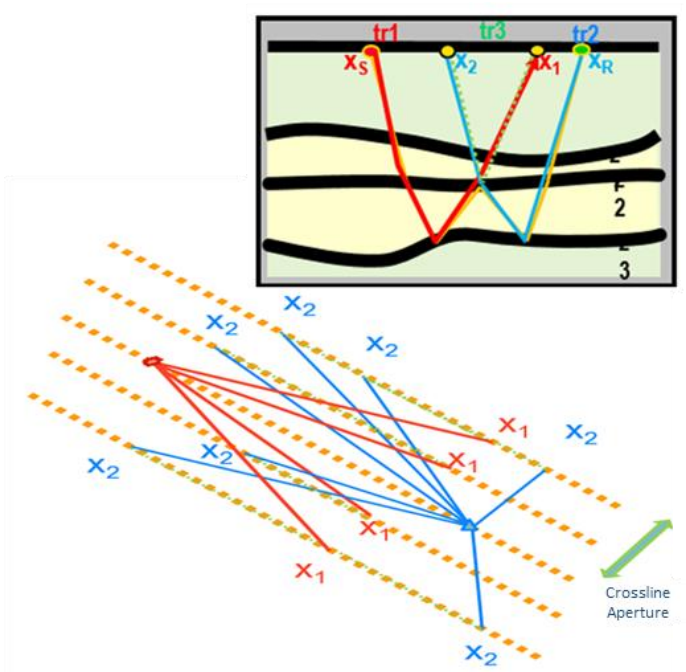

Figure 2 - Linear crossline aperture scheme (from Wu et al., 2019).

Adaptive matching and subtraction of the available internal multiple models applied in the data domain better constrain the velocity updates from RFWI. The subtraction process used a simultaneous subtraction method to subtract all multiple models generated by the internal multiple prediction algorithm (Ala'i and Verschuur, 2003). The output from the subtraction focused on removing internal multiples at pre-salt targets. The resultant data was later used as input to the pre-salt RFWI and the final tomography update.

\section{Final internal multiple attenuation in the imaging domain}

Once the final velocity model was available, we performed the final internal multiple attenuation in the post-imaging space. In this domain, both primaries and internal multiples are better focused, especially short-period internal multiples. Overall signal-to-noise ratio was high. The postimaging subtraction process consisted of a cascaded softthreshold performed in the curvelet-transform space. The application of a curvelet transform represents the recorded data and the multiple model in a multiscale, compact, and semi-sparse sense (Candés and Demanet, 2005). This makes it well-suited for internal multiple attenuation, as it enhances components that are present in both datasets (multiples), while allowing the exclusion of components that only exist in one of the datasets (e.g. primaries) from the matching process. Thus, this lead to better preservation of primaries.

\section{Results}

We applied the proposed workflow to a narrow azimuth towed streamer acquisition, acquired over the Santos Basin, offshore Brazil, with water varying from $1450 \mathrm{~m}$ to $2650 \mathrm{~m}$. The main reservoir lies more than $5000 \mathrm{~m}$ below the seafloor and under a complex overburden composed of structural trend variations, evaporites with variable thickness, and igneous layers mixed with sandstone, 
shale, and salt. Carbonate reservoirs are deep and heterogenous beneath a complex and stratified salt body.

The internal multiple modeling process used the interfacebased method over a layered generating sequence, starting immediately below the seafloor, and until and slightly below the base of salt. Each generating interval was $400 \mathrm{~ms}$ in time, covering all the possible internal multiples arriving at the target zone. The internal multiple prediction process used a variable aperture and DRP sampling scheme along with an optimized selection of the triplets.

Figure 3 shows a comparison over two distinct commonchannel gathers (near- and far-offset) between the recorded seismic, combined internal multiple model, and the adaptive matching in the data domain. The overall model is kinematically accurate across the entire cable length, even in the presence of complex geological features. The adaptive subtraction derives matching filters using a least-squares objective function, providing effective attenuation for intra- and pre-salt layers. Estimated primaries after adaptive matching were used as input to the RFWI process.

RFWI velocity updates applied in this seismic experiment focused on intra- and pre-salt intervals. We performed a total of 5 RFWI iterations for each update, with updates alternating between salt and pre-salt, and pre-salt only layers. A comparison between the velocity field and delta velocity (DV) attributes of the RFWI updates were performed on observed data without and with internal multiple attenuation (Figure 4). The display shows the positive impact of the internal multiple attenuation process, preventing unrealistic updates that would slow down the velocity field and incorrectly position the base of the salt and pre-salt layers.

Final IMA results are shown in Figure 5, showing a Kirchhoff Pre-stack Depth seismic image obtained with the updated velocity model with three versions of data migrated: before separation, the estimated primaries, and the estimated internal multiples. The highlighted segment of the picture shows the main impact of the IMA workflow, removing the coherent interference contaminating the formations below the base of salt. IMA improves interpretability of the key pre-salt events, providing a more confident input to the process of delineating a potential prospect.
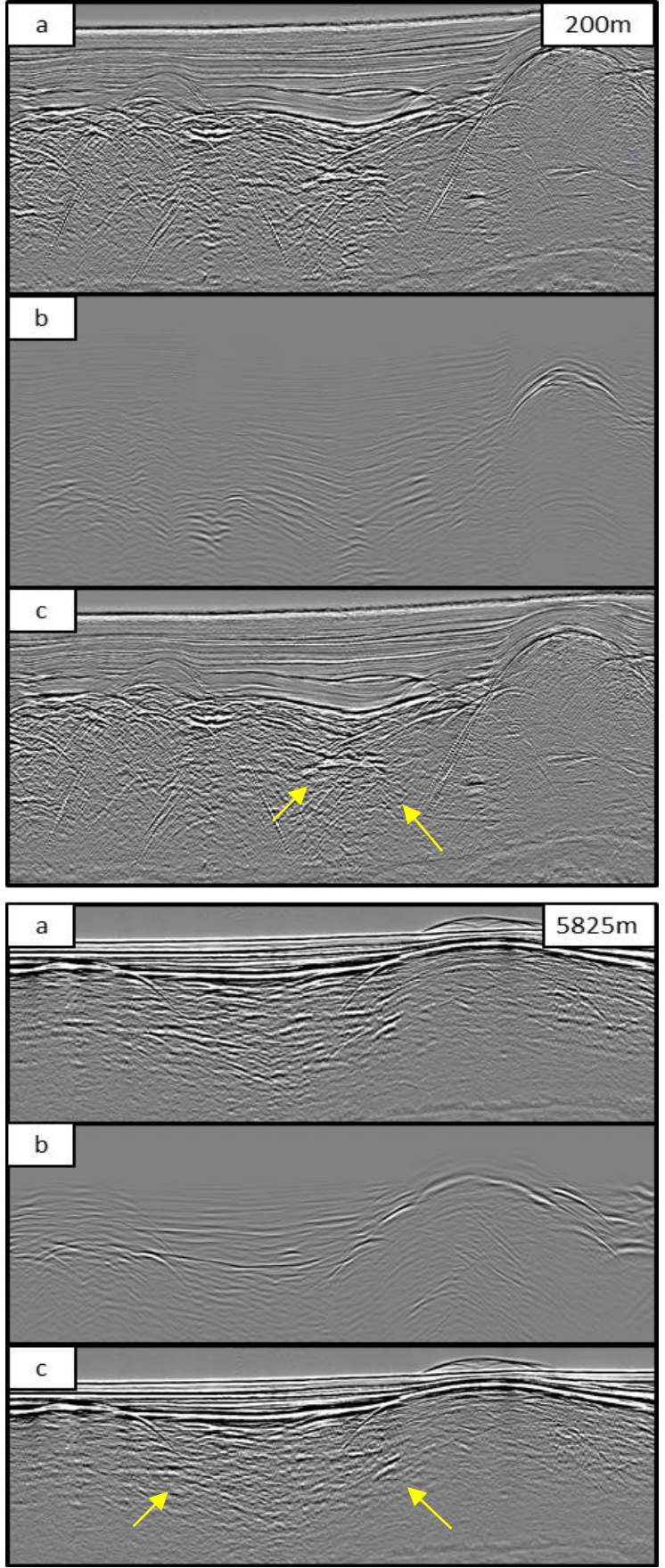

Figure 3 - Input seismic, predicted model, and adaptive subtraction in data domain. Common trace gathers at offset $200 \mathrm{~m}$ (top) and $5825 \mathrm{~m}$ (bottom). Highlighted area shows the input (a), after internal multiple attenuation (b) and the estimated internal multiples (c). Yellow arrows show locations where strong internal multiples were successfully attenuated. The data after the subtraction (c) was used as input to RFWI. 

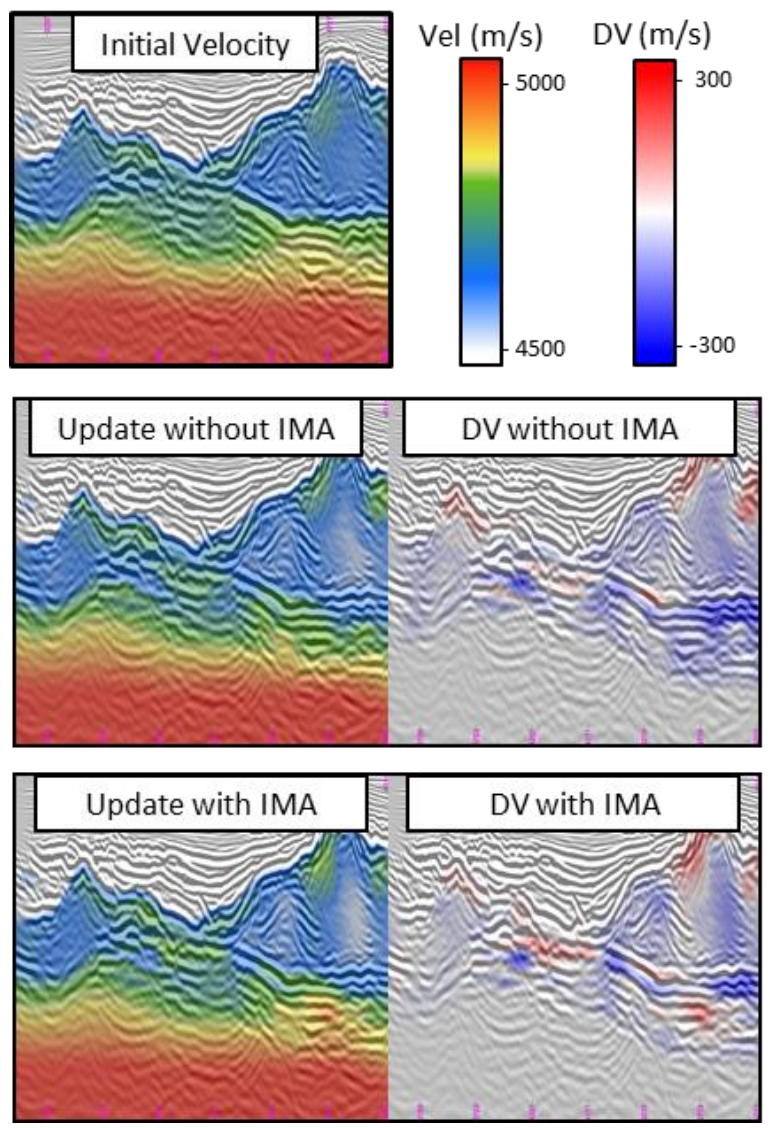

Figure 4 - Comparison between the initial velocity model (above) with RFWI updates shows how internal multiples can interfere with the velocity model building process. Model generated with input data without (middle) and with IMA (below) showed subtle but important differences at the pre-salt layers (as displayed by DV attributes).

\section{Conclusions}

Internal multiple attenuation is a key process when looking at deep reservoir targets under a complex and highly stratified overburden. Data-driven IMA methods are the recommended approach to reduce ambiguities in the velocity model-building process and improve the final imaging outcome. This work proposed a three-pronged strategy to achieve an optimized 3D data-driven workflow, where attenuation of internal multiples served the purpose of reducing the velocity model-building inaccuracies while improving the final imaging outcome at the pre-salt layers. This case study conducted in the Santos Basin showed a positive outcome when applying the proposed workflow, setting the correct kinematic model and improving the interpretability of events placed below the complex salt structure.

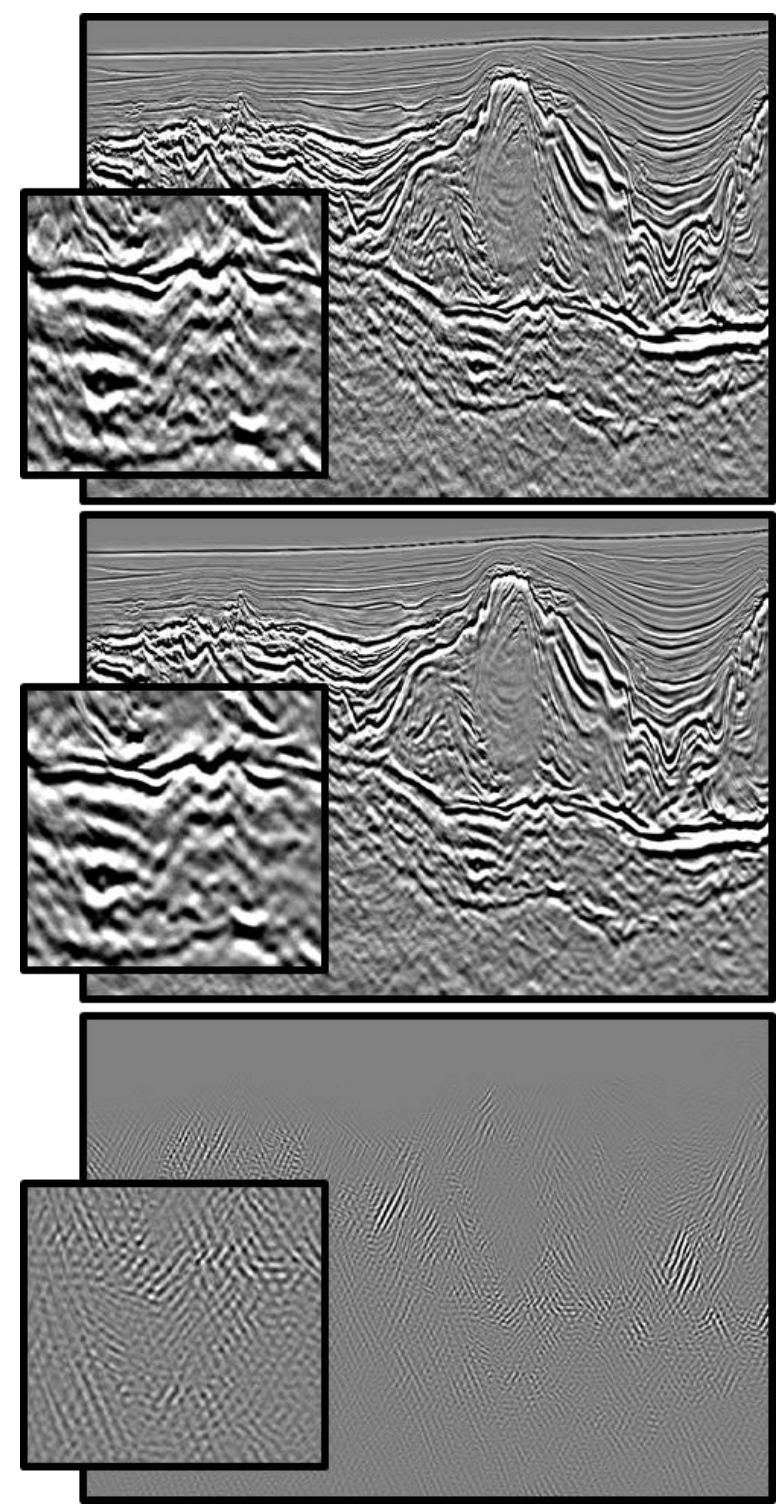

Figure 5 - Input image (above), estimated primaries (middle) and estimated internal multiples (below). Highlighted area shows the removal of the coherent interference contaminating the formations below the base of salt, improving the imaging quality.

\section{Acknowledgments}

We thank the Libra Consortium for granting permission to publish this work.

\section{References}

Ala'i R., D.J. Verschuur 2003 Simultaneous adaptive leastsquares subtraction of multiples. 65th EAGE Conference \& Exhibition, Extended Abstracts, P193.

Candés, E.J., and L. Demanet, 2005, The curvelet representation of wave propagators is optimally sparse, Comm. Pure Appl. Math, 58-11: 1472-1528. 
Jakubowicz, H., 1998, Wave equation prediction and removal of interbed multiples, SEG Technical Program Expanded Abstracts, 1527-1530.

Sun, D., K. Jiao, X. Cheng, and D. Vigh, 2018, Keys to robust reflection-based full-waveform inversion, SEG Technical Program Expanded Abstracts, 1283-1287.

van der Neut, J., and K. Wapenaar, 2016, Adaptive overburden elimination with the multidimensional Marchenko equation, Geophysics, 81, T265-T284.

Verschuur, D. J., 2006, Seismic multiple removal techniques: Education tour series, EAGE.

Weglein, A.B., F.A. Gasparotto, P.M. Carvalho, and R.H. Stolt, 1997, An inverse-scattering series method for attenuating multiples in seismic reflection data, Geophysics, 62, 1975-1989.

Wu, Z. J., S. Sonika, and W. Dragoset, 2011, Robust Internal Multiple Prediction Algorithm: 81st Annual International Meeting, SEG, Expanded Abstracts, 35463549.

Wu, Z.J., A.R El Zein, F. Xavier de Melo, and C. Kostov, 2019, Reflection seismology internal multiple estimation, WO 2019/010253 Al1, published 10-Jan-2019. 